\title{
The cortical distribution pattern of diabetic glomerulopathy
}

\author{
A. Hørlyck, H.J.G. Gundersen and R.Østerby \\ Stereologic and Electron Microscopic Laboratory for Diabetes Research, University Institute of Pathology and 2nd University Clinic \\ of Internal Medicine, Institute for Experimental Clinical Research, Aarhus University, Aarhus, Denmark
}

\begin{abstract}
Summary. In advanced diabetic nephropathy, all nephrons are affected. However, glomerulopathy shows some variation in severity. The aim of this study was to see whether the distribution of glomerular lesions bears any relationship to known renal anatomical/functional organization. Autopsy material from 12 long-term Type 1 (insulin-dependent) diabetic patients was collected retrospectively. Sections presenting the whole depth of cortex were subdivided into 3 zones of equal width. Two parameters were estimated in the deep and the superficial zones separately: (1) in open glomeruli, the volume of red-stained (PAS-positive) material as a fraction of the total glomerular volume, and (2) the number of occluded glomeruli as a fraction of the total number. The results show that the volume fraction of red-stained (PAS-positive) material, as well as the frequency of occluded glomeruli, are similar in the superficial and the deep zone. In addition, the spatial distribution
\end{abstract}

of occluded glomeruli was tested for randomness. The occluded glomeruli are not randomly distributed, but show clustering. Furthermore, they tend to be located in columns perpendicular to the kidney surface, a tendency which cannot be explained by the general tendency for glomeruli to show such an arrangement. We conclude that it is unlikely that the combined structural and functional differences that exist between the superficial and deep glomeruli play a major role in the development of diabetic glomerulopathy. The kidney topology influences the process of glomerular occlusion. Several a priori, plausible mechanisms for the process are discussed.

Key words: Diabetic nephropathy, diabetic glomerulopathy, long-term diabetes, glomerular distribution, glomerular occlusion, morphometry, pattern analysis, pattern distribution, point patterns.
Diabetic glomerulopathy causes clinical nephropathy, and eventually end-stage renal disease, in a substantial proportion of long-term Type 1 (insulin-dependent) diabetic patients. Although all nephrons are involved, the severity of the lesion varies between glomeruli. Some are totally occluded, and the remaining ones show varying sizes and varying amounts of solid material relative to luminal spaces.

The aim of this study was to see whether the distribution of the lesions bears any relationship to renal anatomical/functional organization. Systematic differences are known to exist between layers parallel to the cortical surface, i. e. between superficial and deep (juxtamedullary) nephrons. The latter are larger [1,2], and, in experimental animals, they reportedly have a higher glomerular filtration rate (GFR) and probably a higher intracapillary pressure [3].

Another subgroup of the nephrons which was considered in the present study is that subgroup related to units of glomeruli supplied from the same interlobular artery, i. e. columns perpendicular to the kidney surface.
If diabetic glomerulopathy exhibits a pattern within the kidney related to known structural/functional organization, this may point to possible mechanisms operating in its development.

\section{Materials and methods}

\section{Materials}

Autopsy material from long-term Type 1 diabetic patients was collected retrospectively, disregarding the causes of death. All patients fulfilled a set of inclusion criteria: 1) they had classical insulin-dependent diabetes of more than 15 years duration (average 32 years), with onset of the disease before the age of 40,2 ) the blocks of kidney tissue should include the whole depth of cortex from capsule to medulla, and 3) unquestionable diabetic glomerulopathy should be present. From each block of kidney tissue, new sections were cut and stained with PAS.

Twelve patients were selected for study, six women and six men, aged 28-71 years (average age 53). In terms of clinical nephropathy, which was not a criterion for inclusion in the study, the whole spectrum was covered: from normal kidncy function (albustix-negative urine and normal serum creatinine) to death in uraemia. 


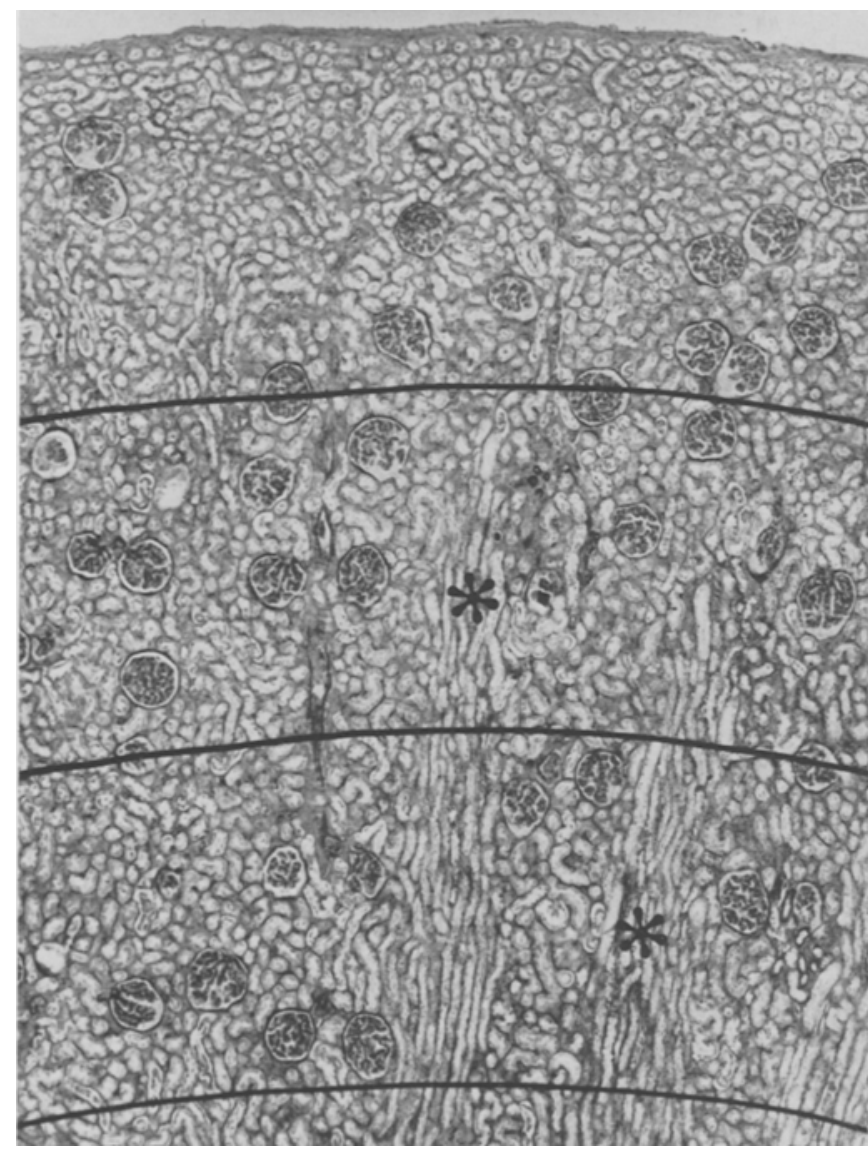

Fig. 1. Section of kidney cortex with renal capsule above and the cortex-medulla transition at the bottom. The three 'parallel' zones are marked off. A few medullary rays are seen (asterisks), demonstrating a tendency toward an arrangement of the glomeruli perpendicular to the kidney surface

\section{Methods}

The kidney cortex was subdivided into three zones of equal width by drawing lines directly on the cover slips which represented a superficial, an intermediate and a juxtamedullary zone (Fig. 1). Two different parameters were chosen as expressions of the zonal severity of diabetic glomerulopathy: 1) in open glomeruli, the volume of red-stained material as a fraction of the total glomerular volume, and 2) the number of occluded glomeruli as a fraction of the total number. A glomerulus is categorized as occluded if no open capillaries are seen in the section. The term 'glomerulus' is used synonymously with 'glomerular profile', defined as the glomerular tuft itself excluding the capsule of Bowman and the capsular space. In the evaluation of zones parallel to the kidney surface only the superficial and the deep zones were used.

The volume fraction of red-stained material was obtained only from a total of about 35 open glomeruli in each zone. Measurements were performed with the field of vision projected onto the table at a final magnification of $1053 \times$ using a Zeiss $40 / 0.65$ objective. Open glomeruli were sampled by moving the visual field along two parallel lines as illustrated in Fig. 2 [4]. The distance between the sampled stripes was determined by the approximate number of open glomeruli. The volume fraction of red-stained (PAS-positive) material to total glomerular volume, $V_{V}$ (Red/Glom), was determined by point counting using the test system shown in Fig. 3. The relative number of occluded glomeruli was estimated by simply counting all the open and occluded glomerular profiles in each zone. On the average, 375 glomeruli were counted in each patient. The absolute percentage given for glomerular occlusion are underestimations due to the fact that occluded glomeruli have a smaller diameter than the open ones [5], and

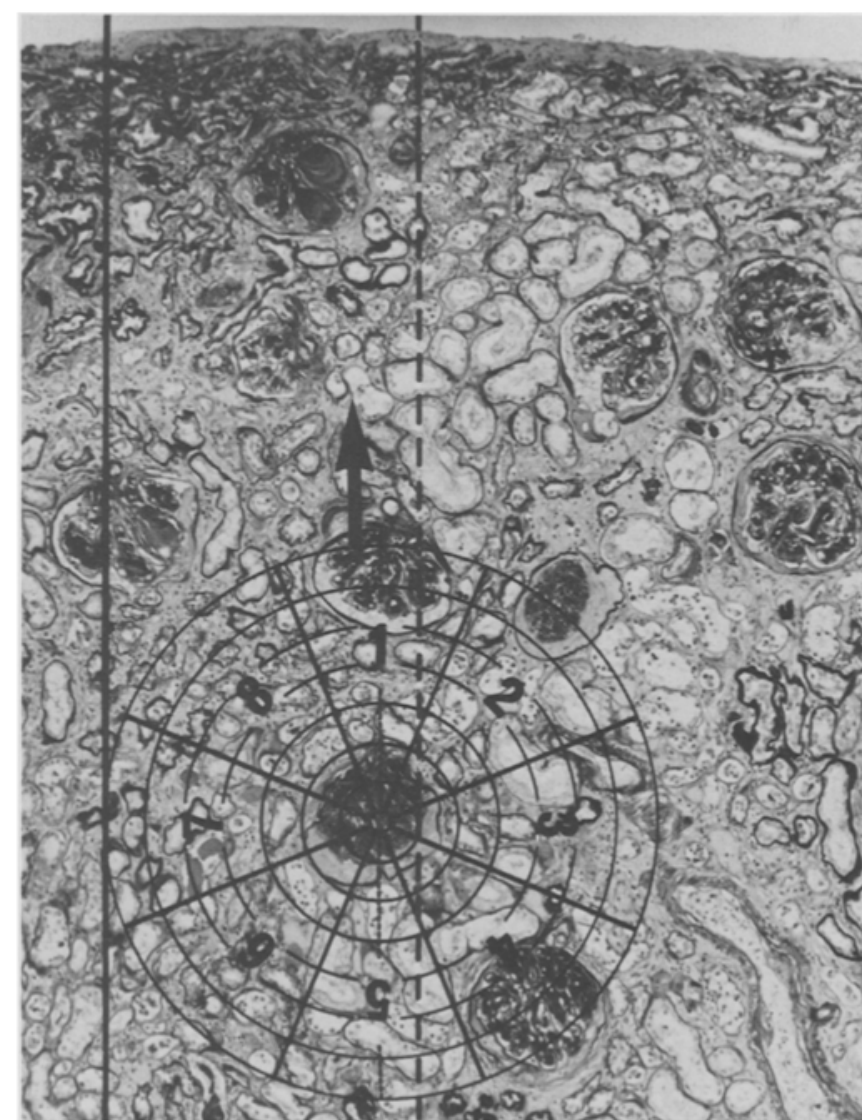

Fig. 2. Kidney cortex with the sampling lines along which the visual fields were moved. Glomeruli between the lines or touching the righthand line were sampled, and those touching the left-hand line were excluded to give an unbiased sample [4]. The circular grid divided into sectors was used for analysing the neighbour relations between glomeruli and the glomerular occlusion pattern. The test system was centered on an occluded glomerulus with the arrow perpendicular to the kidney surface. The nearest neighbour was considered to be in a perpendicular direction if its center was in sector 1 or 5 , and in a parallel direction if its center was in sector 3 or 7 . If the nearest neighbour was in an oblique direction, i.e. in an even numbered sector, the central glomerulus was excluded from the directional analyses

small glomeruli have a smaller chance of being hit by the section than large glomeruli [6]. This does not, of course, influence the detection of gradients or patterns in glomerular occlusion.

Finally, observations were made to test whether a pattern of glomerular occlusion was detectable. One case (no.7) was omitted from this analysis because only 7 occluded glomeruli were present. The analysis is performed in two steps, the first being a search for whether there is non-randomness in the 2-dimensional arrangement of occluded glomerular profiles. If the arrangement is not random by the criterion used, one can then look, in the second step, for certain specified classes of patterns. (For a general account of analysis of point patterns, see the recent book by Diggle [7]).

A rather simple analysis was used to test for non-randomness: the relationship between nearest neighbour. If the relationship between occluded glomeruli were random, the nearest neighbour to an occluded glomerulus would itself be occluded with a frequency equal to the average, overall frequency of occlusion among glomeruli. Two general classes of non-random relations at the level of individual glomeruli are detectable with this analysis: clustered and systematic. In clustered arrangements, occluded glomeruli have a larger than average frequency of occluded neighbours; in systematic arrangements, 


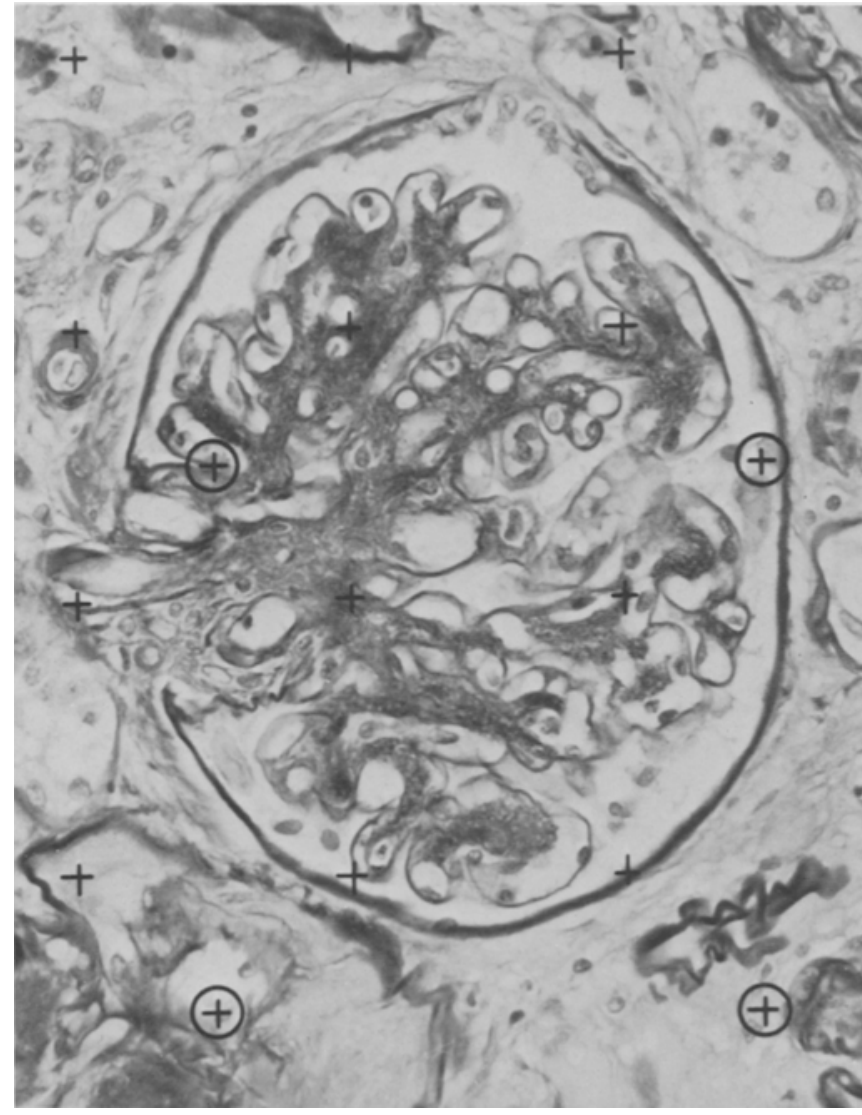

Fig. 3. Glomerular profile with test system superimposed. The grid with two sets of points (coarse and fine, 1:4) was used to determine the volume fraction of red stained material with the glomerular tuft as the reference volume

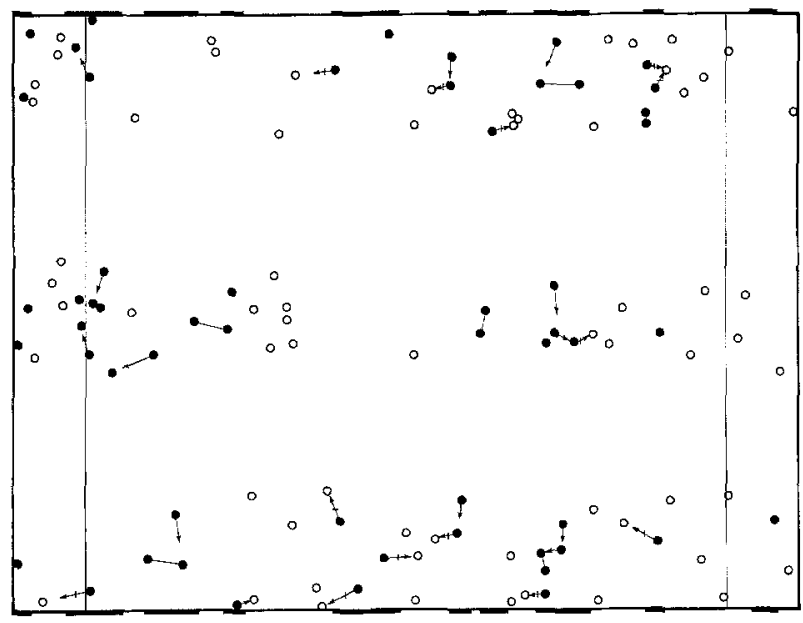

Fig.4. Simulation of kidney cortex with a non-anatomical arrangement of open $(0)$ and occluded $(\bullet)$ glomeruli placed at random within 3 zones parallel to the kidney surface (top). Glomerular occlusion is solely in stripes perpendicular to the kidney surface (corresponding to the heavy bars). The results of an analysis of the directional pattern are given in the text

occluded and open glomeruli tend to alternate in some systematic way leading to a lower than average frequency of occlusion among nearest neighbours to occluded glomeruli.

As shown below, we found an arrangement of clustered occlusion among glomeruli. Obviously there is an infinite number of possible
V (RED / GLOM)

FRACTION OF GLOMERULI OCCLUDED
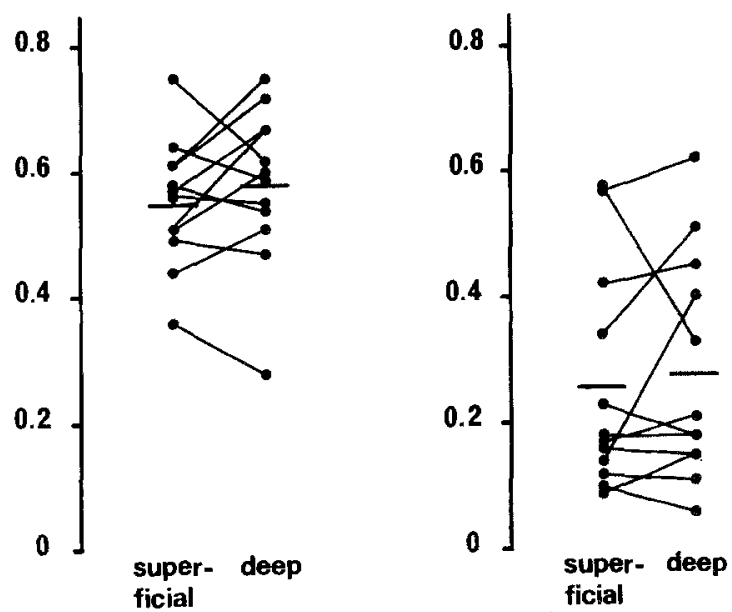

Fig.5. The two parameters of diabetic glomerulopathy compared in superficial and deep zones. Results in individual diabetic patients are connected. Group means are shown as horizontal bars

patterns in which the clusters can be arranged. We were interested in a possible relationship to the known anatomical arrangement of glomeruli in units perpendicular to the kidney surface, and we therefore in the next step of the analysis designed a test for such a subclass of cluster patterns: 'stars in stripes'.

Two sets of observations were made for all occluded glomeruli (or a systematic subsample thereof): 1) the direction in which the nearest neighbour is found, parallel or perpendicular to the kidney surface, and 2) the status of this nearest neighbour, occluded or open. For this analysis, the test system shown in Fig. 2 was centered on each occluded glomerulus and the 'vertical' direction in it was aligned perpendicularly to the trace of the kidney surface.

As seen from an occluded glomerulus, the overall ratio between nearest neighbours of any status found in directions perpendicular and parallel to the kidney surface will depend on the glomerular pattern in the section. (As shown in column A in Table 2, we found the expected preponderance for neighbours to a given glomerulus to be in perpendicular, rather than parallel directions). The pattern of glomerular occlusion is, however, revealed by noticing in the second set of observations the ratio between perpendicular- and parallel-aligned nearest neighbours to occluded glomeruli which are themselves occluded (Table 2). If the alignment ratio differs from the general ratio, the occlusion pattern does not have a random direction. Since the anatomical arrangement of glomeruli in the sections is to some extent in stripes perpendicular to the kidney surface, it is important that the test for an occlusion pattern is essentially independent of the underlying general pattern.

\section{Synthetic example}

In order to illustrate that the applied statistical parameter of the occlusion pattern is independent of the anatomical arrangement, an artificial section of kidney cortex was made (Fig. 4). The glomeruli have been distributed with a random position within 3 zones parallel to the kidney surface. A process occluding all glomeruli has been at play within vertical stripes, the limits of which are selected at random. For each occluded glomerulus, the direction of its nearest neighbour is found using the test system shown in Fig. 2. Occluded glomeruli are not sampled within the two rows outside the sampling field (indicated by two thin vertical lines) because such glomeruli may have nearest neighbours outside the edge of the block. Each arrow points from an occluded glomerulus within the sampling field to its nearest neighbour. Either of two occluded glomeruli joined by a line has the other as its nearest neighbour. Only neighbouring relations in directions 
perpendicular or parallel to the kidney surface are considered, with oblique directions as defined by the sectors in the test system being excluded. Of the 46 occluded glomeruli within the sampling field, 8 have nearest neighbours in oblique directions and are given no arrows, 21 have their nearest neighbours parallel to the kidney surface and 17 have them perpendicular to the surface. If the nearest neighbour to an occluded glomerulus is open the arrow is transversed by a bar. Nine out of 21 parallel neighbour relations are occluded-to-occiuded ( $43 \%$ ), whereas 15 of 17 perpendicular relations are occludedto-occluded $(88 \%)$. There is thus a higher frequency of parallel than perpendicular neighbour relations to occluded glomeruli. This reflects the (non-anatomical) arrangement of glomeruli in rows parallel to the kidney surface. Despite this arrangement, there are only 9 parallel as opposed to 15 perpendicular occluded-to-occluded neighbour relations, reflecting that the occlusion process is confined to perpendicular stripes.

Table 1. Neighbour relations of occluded glomeruli

\begin{tabular}{|c|c|c|c|}
\hline Patient no. & Frequency $(\%)$ & $\begin{array}{l}\text { Frequency of } \\
\text { nearest neighbour } \\
\text { occluded }(\%)\end{array}$ & $\begin{array}{l}\text { Difference } \\
\text { in frequency }\end{array}$ \\
\hline 1 & 25 & 50 & 25 \\
\hline 2 & 47 & 56 & 9 \\
\hline 3 & 12 & 26 & 14 \\
\hline 4 & 61 & 68 & 7 \\
\hline 5 & 17 & 71 & 54 \\
\hline 6 & 12 & 27 & 15 \\
\hline 8 & 13 & 33 & 20 \\
\hline 9 & 40 & 39 & -1 \\
\hline 10 & 16 & 22 & 6 \\
\hline 11 & 17 & 48 & 31 \\
\hline \multirow[t]{2}{*}{12} & 45 & 69 & 24 \\
\hline & & $\begin{array}{l}\text { Mean } \\
\text { SEM }\end{array}$ & $\begin{array}{r}18.5 \\
4.6\end{array}$ \\
\hline
\end{tabular}

The overall frequencies of occluded glomeruli in individual patients are shown in the second column. The third column shows the frequency with which the nearest neighbour to an occluded glomerulus is itself occluded

\section{Statistics}

Intra-individual differences were judged by Student's paired t-test, and a $2 p$-value $<0.05$ was considered significant. The ratios A and B in Table 2 were compared after logarithmic transformation due to the rather right-skewed sample distribution of their ratio.

\section{Results}

The two parameters expressing the severity of diabetic glomerulopathy in the superficial and deep zones, respectively, are shown in Fig. 5; there was no tendency towards a preferential occurrence in either one of the two zones.

Table 1 shows the results of the first part of the analysis of the distribution of glomerular occlusion. In 10 of 11 cases, the nearest neighbour to an occluded glomerulus was occluded itself with a frequency higher than the average frequency $(2 p=0.0023)$, i.e. the clustering of occluded glomeruli is evident.

Table 2 gives the result of the directional analysis, in which the anatomical distribution of glomerular profiles (as revealed in column A) is taken into account. The right-most column shows that, in 10 out of 11 cases, the nearest neighbour - when occluded itself - is in the perpendicular direction more frequently than expected $(2 p=0.0042)$, indicating that the occlusion process works with some preponderance for stripes perpendicular to the kidney surface.

\section{Discussion}

The results from the first part of the investigation show that there is no difference in the severity of glomerulopathy between the deep and the superficial glomeruli.

Table 2. Analysis of the directions to nearest neighbour from occluded glomerulus

\begin{tabular}{|c|c|c|c|c|c|c|c|}
\hline \multirow{2}{*}{$\begin{array}{l}\text { Patient } \\
\text { no. }\end{array}$} & \multicolumn{3}{|c|}{ Nearest neighbour open or occluded } & \multicolumn{4}{|c|}{ Nearest neighbour occluded } \\
\hline & $\begin{array}{l}\text { No. } \\
\text { perpendicular }\end{array}$ & $\begin{array}{l}\text { No. } \\
\text { parallel }\end{array}$ & $\begin{array}{l}\text { A } \\
\text { Ratio } \frac{\text { perp. }}{\text { parallel }}\end{array}$ & $\begin{array}{l}\text { No. } \\
\text { perpendicular }\end{array}$ & $\begin{array}{l}\text { No. } \\
\text { parallel }\end{array}$ & $\begin{array}{l}\text { B } \\
\text { Ratio } \frac{\text { perp. }}{\text { parallel }}\end{array}$ & $\frac{B}{A}$ \\
\hline 1 & 39 & 24 & 1.63 & 18 & 13 & 1.38 & 0.852 \\
\hline 2 & 40 & 28 & 1.43 & 28 & 13 & 2.15 & 1.508 \\
\hline 3 & 24 & 43 & 0.56 & 6 & 7 & 0.86 & 1.536 \\
\hline 4 & 29 & 32 & 0.91 & 22 & 19 & 1.16 & 1.278 \\
\hline 5 & 38 & 27 & 1.41 & 28 & 18 & 1.56 & 1.105 \\
\hline 6 & 23 & 22 & 1.05 & 8 & 4 & 2.00 & 1.913 \\
\hline 8 & 24 & 21 & 1.14 & 10 & 6 & 1.67 & 1.458 \\
\hline 9 & 32 & 29 & 1.10 & 16 & 12 & 1.33 & 1.208 \\
\hline 10 & 40 & 33 & 1.21 & 13 & 4 & 3.25 & 2.681 \\
\hline 11 & 30 & 26 & 1.15 & 17 & 6 & 2.83 & 2.456 \\
\hline 12 & 37 & 31 & 1.19 & 27 & 20 & 1.35 & 1.131 \\
\hline Geomet & & & 1.12 & & & 1.65 & 1.47 \\
\hline
\end{tabular}

The second and the third columns show - with respect to occluded glomeruli - the number of nearest neighbours which are in a direction perpendicular or parallel to the kidney surface. The column marked " $\mathrm{A}$ " is the ratios of these numbers. For this part of the analysis the nearest neighbour was taken into account irrespective of its status (open/occluded). In the right-hand part is shown the corresponding outcome in which only occluded nearest neighbours were analysed. The last column shows the 'perpendicularity' of occluded glomeruli as opposed to that of open and occluded glomeruli. The geometric mean ratios are shown below 
This indicates that none of the existing structural/functional differences between the superficial and deep glomeruli plays a major role in the development of the glomerulopathy. The data presented here do not, however, exclude that a high pressure gradient across the capillary wall may be a contributing factor in the progression of diabetic glomerulopathy. A similarly uniform distribution among the 'parallel' zones of kidney cortex of the glomerular occlusion occurring as an aging phenomenon has previously been reported [8].

Expectedly, the estimates of $V_{V}$ (Red/Glom) in open glomeruli and the fraction of occluded glomeruli showed no consistent relationship in individual patients.

The study of the distribution of the glomerular occlusion was only conducted at a rather low level, asking only simple questions. Whereas it is quite easy to detect and statistically test for non-randomness, the alternative - a pattern of the clusters - has an infinite number of possibilities. For the second step in the analysis, we have therefore used an ad hoc designed test, the positive outcome of which is only compatible with a certain pattern. The existence of any given pattern cannot, of course, be proven in the ordinary sense. It should moreover be noted that the analysis is only made on two-dimensional sections - for rather obvious reasons of cost and work-load. The conclusions regarding the underlying three-dimensional structure - glomeruli distributed in the cortex - and the temporal and dynamic occluding process(es) cannot therefore be more than tentative: the process seems to be at play in columns perpendicular to the cortical surface rather than at random or in layers parallel to the surface.

A pattern in the glomerular occlusion in the diabetic kidney has not previously been studied, and it is as yet unknown whether a similar pattern exists in the distribution of early diabetic glomerulopathy in open glomeruli. Only tentative guesses can be made concerning factors that may cause the clustering in columns. Part of the explanation may be that, once a glomerulus is occluded, it will secondarily affect the neighbouring glomeruli. This could come about by local alterations in blood flow in that particular interlobular artery. One could also imagine that consequences of stopping efferent arteriolar flow, i.e. blood supply for the tubular system of neighbouring nephrons and the interstitium, would be likely to show up in columns perpendicular to the kidney surface due to the orientation of the tubular system. Ischaemic changes in the distal part of the nephron and/or the interstitium may then eventually affect the glomeruli in question. The tendency for glomerular occlusion to occur in columns perpendicular to the kidney surface may also point to possible primary factors related to the interlobular arteries. A locally reduced blood flow due to diabetic macroangiopathy in these vessels could play a role. Quite a different disease process, interstitial nephritis, should also be considered. It has been shown that significant bacteriuria occurs with increased frequency in diabetic patients [9] and an 'interstitial nephritis' is not an uncommon finding in diabetic patients [10]. In two patients in the present series (cases no. 2 and 3), a very marked acute inflammatory process was ongoing with papillary necrosis, and in a further patient (case no.5) there was a rather marked histologically defined pyelonephritis. However, as shown in Table 2, these cases did not demonstrate any particularly high degree of column arrangement.

Glomerular occlusion constitutes a very significant aspect of advanced diabetic glomerulopathy. Consequences in terms of loss of renal function are immediately comprehensible, since compensatory enlargement of the remaining glomeruli cannot prevent the inevitable outcome [5]. The major fundamental cause in all likelihood is the continued accumulation of basement membrane material characteristic of diabetic glomerulopathy. It seems, however, that we still need more information to understand the mechanisms involved in these processes. We also need to learn more about the events that take place in the remaining still open and functioning glomeruli at this stage of development.

Acknowledgements. Mr. A. Meyer and Ms. A. Larsen are thanked for making the illustrations. The study was supported by grants from the P. Carl Petersen Fond and University of Aarhus.

\section{References}

1. Sørensen FH (1972) Quantitative studies of the renal corpuscles I: intraglomerular, interglomerular and interfocal variation in the normal kidney. Acta path microbiol scand Section A 80: 115-124

2. Olivetti G, Anversa P, Melissari M, Loud AV (1980) Morphometry of the renal corpuscle during postnatal growth and compensatory hypertrophy. Kidney Int 17: 438-454

3. Müller-Suur R, Ulfendahl HR, Persson AEG (1983) Evidence for tubulo-glomerular feedback in juxtamedullary nephrons of young rats. Am J Physiol 244: F425-F431

4. Gundersen HJG (1977) Notes on the estimation of the numerical density of arbitrary profiles: the edge effect. J Microsc 111: 219-223

5. Gundersen HJG, Østerby R (1977) Glomerular size and structure in diabetes mellitus. II Late abnormalities. Diabetologia 13:43-48

6. Weibel ER (1979) Stereological methods, vol I, Practical methods for biological morphometry. Academic Press, London New York Toronto

7. Diggle PJ (1983) Statistical analysis of spatial point patterns. Academic Press, London

8. Kaplan C, Pasternack B, Shah H, Gallo G (1975) Age-related incidence of sclerotic glomeruli in human kidneys. Am J Pathol 80: 227-234

9. Vejlsgaard R (1966) Studies on urinary infection in diabetics. II. Significant bacteriuria in relation to long-term diabetic manifestations. Acta Med Scand 179: 183-188

10. Ditscherlein G (1969) Nierenveränderungen bei Diabetikern. Fischer, Jena

Received: 22 July 1985

and in revised form: 6 December 1985

Arne Hørlyck

Diabetes Laboratory

Bartholin Building

University of Aarhus

DK-8000 Aarhus C

Denmark 